RENSSELAER POLYTECHNIC INSTITUTE

TROY, NEW YORK 12180-3590

$\begin{array}{ll}\text { TECHNICAL PROGRESS REPORT } & \begin{array}{l}\text { DOE/ER/53211--10 } \\ \text { DE92 } 005299\end{array}\end{array}$

(Covering the period October 1, 1990 through

December 15, 1991)

under

DOE GRANT NO. DE-FG02-85ER53211.A002

entitled

\title{
ADVANCED DEVELOPMENT OF PARTICLE BEAM PROBE DIAGNOSTIC SYSTEMS
}

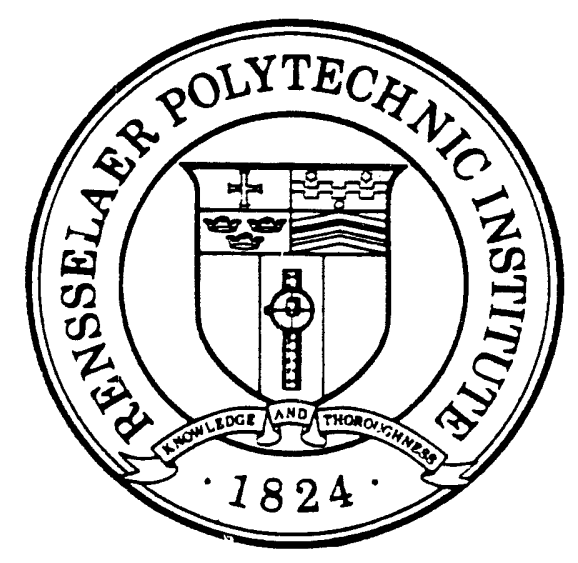

\section{Prepared by}

R. L. Hickok, T. P. Crowley, K. A. Connor and P. M.

Schoch

December 1991 


\section{PROGRESS REPORT}

This progress report under DOE Grant DE-FG02-85ER3211 covers the period October 1, 1990 through December 15, 1991. During this period the co-principal investigators, R. L. Hickok and T. P. Crowley each spent approximately $50 \%$ of their time on this project.

During the period covered by this report the $2 \mathrm{MeV}$ heavy ion beam probe (HIBP) was installed on the TEXt tokamak. This system is now operational at reduced voltages $(<750 \mathrm{keV})$ using the $100 \mathrm{kV}$ electrostatic energy analyzer from the previous $500 \mathrm{keV}$ HIBP. The analyzer was modified to eliminate the guard rings by installing a shaped top plate and the gain was increased from 5 to 6 . The first secondary signals were obtained in early December and the system is undergoing final alignment and resting at the present time. The initial results showed an order of magnitude increase in UV generated currents on the split plate detectors over that observed with the $500 \mathrm{keV}$ beam probe system. We had expected an increase due to the replacement of the wire screen top plate with a solid top plate, but the increase was larger than anticipated. There was also a large increase in the UV generated current to the top plate. Closing the entrance slits to the analyzer reduced the UV generated currents on the split plate detectors to essentially zero but only reduced the top plate current by about $20 \%$. Closing the gate valve in the secondary beam line did reduce the top plate current to zero. From this we conclude that the split plate currents are due to UV that is coming through the entrance slits and being reflected by the top plate onto the detectors. The majority of the top plate current appears to be due to UV that is entering the analyzer chamber by means other than the entrance slits. 
This simply means that we need to improve the UV baffling around the entrance port to the analyzer chamber. We probably will not do this for the present setup, but we will design the $400 \mathrm{kV}$ analyzer system so that the only way UV can get into the analyzer chamber is through the entrance slits. The L'V current on the split plates is primarily more of a nuisance than a serious problem for most of the measurements we want to make. It is slowly varying current over the plasma pulse and behaves like a DC offset. If we subtract this offset then the best signal to noise ratio we observed on these preliminary measurements was about 50 to 1 . This UV current does reduce the dynamic range that we have available and it could be troublesome when we try to look at changes in the plasma that are driven for example, by turning on the $\mathrm{ECH}$ since this may also change the UV emission, consequently we are looking at ways of reducing the UV generated split plate currents. We are still working our way up the learning cunse on the operation of this accelerator and most of the system is still under manual control. We expect to slowly bring the system under computer control next year. As is however, the system should be capable of carrying out fluctuation measurements and we expect to be taking data with this system in early 1992.

The $400 \mathrm{keV}$ energy analyzer that will permit HIBP operation at the full $2 \mathrm{MeV}$ energy is still under test at RPI. As expected this is the pacing item in obtaining full 2 $\mathrm{MeV}$ uperation. A full scale aluminum model of the $400 \mathrm{kV}$ analyzer has been tested at $365 \mathrm{kV}$ which is higher than the $335 \mathrm{kV}$ needed for $2 \mathrm{MeV}$ operation. Considerable difficulty was encountered in bringing this large energy analyzer up to operating voltage. Details of these problems and the solutions are contained in the weekly and monthly progress reports that are submitted to R. Mcknight and D. Markevich and will not be 
repeated. ite. We are now convinced that it will be possible to fabricate a parallel plate electrostatic energy analyzer for use with the $2 \mathrm{MeV}$ beam probe system. We also built and tested a $127^{\circ}$ curved plate energy analyzer which was the backup design if tine parallel plate analyzer couldn't be brought up to full voltage. The curved plate analyzer was tested on our high voltage test stand and it worked as predicted provided that the fringing fields were properly included in the analysis. No additional work on the curved plate analyzer is planned at the present time and we are going ahead with the fabrication of an operating version of the $400 \mathrm{kV}$ parallel plate energy analyzer.

Detailed design of the complex shaped vacuum tank for the parallel plate analyzer is nearly completed. The conceptual design was carried out $\tau^{+}$RPI and the detailed design and the completion of the working blueprints was sub-contracted to Princeton Plasma Physics Laboratory. The initial design which was completed in the early fall called for some non-standard spherical and toroidal spinnings that would entail substantial setup charges to have fabricated. We determined that by changing the dimensions slightly we could use standard components with a substantial reduction in cost. We specified the necessary dimension changes and requested Bob Walls of PPPL to supply us with a revised set of drawings. We have just received the drawings and are in the process of going over them in detail. We have found a few minor errors that needed to be corrected and we hope to go out for bids in early 1992.

Conceptual design of the vacuum chambers for the secondary beam line that mates to the bottom port on our pie sector on TEXT has been carried out. This is the beam line that will provide the major coverage of the TEXT plasma at $2 \mathrm{MeV}$ operation and is the one that will be installed first. We plan to incorporate both radial and toroidal 
steering plates in the secondary beam line for the first time. If they work as expected it should provide much greater flexibility in operating the beam probe system. There will also be two bellows in the beam line. One near the analyzer tank will permit a $10^{\circ}$ rotation of the analyzer for carrying out in-situ angular correction curves. Bob Walls has incorporated the drive mechanism for this rotation in the analyzer support structure. The second bellows located closer to the tokamak will permit toroidal rotation of the analyzer system. This flexibility is desirable when switching from diverter to limiter operation of the tokamak. There is a considerable difference in the toroidal displacement of the beam between these two operating scenarios and the second bellows insures that we will be able to optimize the alignment for both operating modes. The schedule for installing the $400 \mathrm{kV}$ analyzer and secondary beam line is late summer or early fall of 1992 . The plan is to install during the period that TEXT is shut down for installation of the diverter coil. This schedule will provide full $2 \mathrm{MeV}$ operation on the same time scale of diverter operation on TEXT.

We have built and carried out preliminary test on a simplified version of what we have refereed to as an enhanced $\widetilde{\phi}$ detector. When incorporated in the energy analyzer it will replace our present entrance slit and detector with, in effect, a set of ten entrance slits and detectors having the same total aperture. The simplified version is not mounted on an energy analyzer but is a linear set of wire ribbons that have to be carefully aligned. We are using this to develop an alignment procedure, evaluate the potential for this detector and to determine what problems we will rur into during fabrication and operation. The linear detector is tested by placing it in the beam line on our test stand and then sweeping the beam across the detector while monitoring the transmitted current. In the 
initial test it was discovered that high beam current densities heated and distorted the wires which spoiled the alignment. By reducing the beam current density to a level near but still higher than that expected for secondary beam current, we were able to maintain the alignment and the detector behaved at a level that was over $90 \%$ of what was predicted. We now have a graduate student assigned to this project and she will continue testing the linear detector and will start developing a procedure for installing a detector assembly on the energy analyzer.

We have continued our effort on evaluating the data obtained with the $500 \mathrm{keV}$ beam probe system on TEXT. In the past, we had shown that the toroidal displacement of the beam at the detector could provide information about the magnetic fluctuations in the plasma. This displacement signal has two components. One component is due to the local fluctuation in the magnetic field at the point where the secondary was createc and the second component was due to the integrated effect over the full trajectory of both the primary and secondary beam. If the signal is dominated by the integrated component then it would provide very little information about magnetic fluctuations. Our initia! analysis has indicated that we were not dominated by the integrated component. During the past year we have carried out detailed trajectory calculations to evaluate the measurements carried out on a large amplitude coherent MHD magnetic fluctuation on TEXT. The results confirmed that in the regions where the amplitude of the magnetic fluctuation was large $(0.3<\mathrm{r} / \mathrm{a}<1.0)$ the signal was dominated by the local component and we had a true measure of the magnetic fluctuation. Near the center of the plasma where the amplitude of the magnetic fluctuation was very weak we were dominated by the path integral effects. This demonstrates that we can provide detailed local information. 
- about coherent magnetic fluctuations but it requires considerable data analysis. In the future, we hope to extend this type of measurement to turbulent magnetic fluctuation.

We are continuing to analyze the possible distortion of our k-vector measurements due to beam modulation. Detailed simulation studies using real trajectories and accurate cross-sections show that for low density plasma (this is the condition for most of our measurements) beam modulation effects are negligible. This increases our confidence that the small k-vectors we observe in the interior of the TEXT plasma are real. 
